and CHD. Their study comprised 41,578 Japanese men and women aged 40-59 years, who had not been previously diagnosed with cardiovascular disease or cancer. Participants completed a food frequency questionnaire and were followed up for 11 years.

The investigators found that fish consumption was associated with a reduced risk of CHD (primarily in myocardial infarction and nonfatal coronary events). Furthermore, they found that the risk of CHD was about $40 \%$ lower among people who ate fish eight times per week (average intake $180 \mathrm{~g}$ per day) than among those who consumed fish only once a week (average intake $23 \mathrm{~g}$ per day). The authors conclude that, for middle-aged people, eating a large quantity of fish seems to provide more protection against CHD than does a modest intake, probably through $\mathrm{n} 3$ polyunsaturated fat consumption.

Claire Braybrook

Original article Iso $\mathrm{H}$ et al. (2006) Intake of fish and $\mathrm{n} 3$ fatty acids and risk of coronary heart disease among Japanese: the Japan Public Health Center-Based (JPHC) Study Cohort I. Circulation 113: 195-202

\section{Does excess dosing of antithrombotics increase the risk of major bleeding?}

Given concern about bleeding risk with use of antithrombotic therapy, Alexander et al. investigated whether unintentional excess dosing of adjustable anticoagulants, specifically unfractionated heparin (UFH), low-molecularweight heparin (LMWH), or glycoprotein Ilb/llla inhibitors, affects outcomes of patients with non-ST-segment elevation acute coronary syndromes.

This prospective analysis included 30,136 patients. Excess doses were received by $42 \%$ of patients who were given at least one antithrombotic agent. Factors associated with excess dosing included age $\geq 75$ years, female sex, renal insufficiency and treatment at a hospital with a history of low adherence to guidelines. Patients receiving excess doses of LMWH or glycoprotein Ilb/llla inhibitors were considerably more likely to experience major bleeding than those receiving recommended doses (adjusted odds ratios 1.39 and 1.36, respectively); a slight trend towards increased bleeding in patients receiving excess UFH was also seen (odds ratio
1.08). Most excess dosages were minor, but $9.9 \%$ of patients receiving UFH, $3.1 \%$ receiving $\mathrm{LMWH}$ and $2.1 \%$ receiving a glycoprotein $\mathrm{Ilb} / \mathrm{llla}$ inhibitor received a major excess dose. For all treatments, major bleeding episodes increased in relation to the degree of excess, but even minor excesses were associated with harm, suggesting that safety concerns persist with any degree of excess. Only excess glycoprotein Ilb/llla inhibitor dosing was associated with increased risk of death, but excess dosing of any of the treatments was associated with longer hospital stay.

Despite use of the correct medications, dosing errors increased the risk of bleeding in already vulnerable patients. The authors urge that doses are calculated carefully, to reduce bleeding risk in these patients.

Rebecca Ireland

Original article Alexander KP et al. (2005) Excess dosing of antiplatelet and antithrombin agents in the treatment of non-ST-segment elevation acute coronary syndromes. JAMA 294: $3108-3116$

\section{Oral amiodarone reduces risk of arrhythmia following cardiac surgery}

Atrial tachyarrhythmia, the most frequent complication following cardiac surgery, is associated with adverse outcomes, prolonged hospitalization, and increased costs. The PAPABEAR trial, which investigated the effects of amiodarone in this setting, reports that a 13-day perioperative course of this drug reduces atrial tachyarrhythmias effectively after cardiac surgery.

Patients due to undergo nonemergency CABG, valve replacement or repair (VRR), or both, were randomly assigned oral amiodarone ( $10 \mathrm{mg} / \mathrm{kg}$ daily, $n=299)$ or placebo $(n=302)$, administered from 5-6 days before surgery to 5-6 days after. During the postoperative period significantly fewer atrial tachyarrhythmias (about $50 \%$ less) were recorded in the amiodarone group than in the placebo group, overall (48 patients vs 89 patients) and in all subgroups: age $<65$ years; age $\geq 65$ years; preoperative $\beta$-blockers; no preoperative $\beta$-blockers, CABG only; and VRR surgery with or without CABG $(P<0.05$ for all). Risk of atrial tachyarrhythmia was greatest in the older-age, VRR and no- $\beta$-blocker groups. Eight patients

\section{GLOSSARY}

PAPABEAR

Prophylactic Amiodarone for the Prevention of

Arrhythmias that Begin Early After Revascularization,

Valve Replacement, or

Repair 